\title{
Structure and polymer form of poly-3-hydroxyalkanoates produced by Pseudomonas oleovorans grown with mixture of sodium octanoate/undecylenic acid and sodium octanoate/5-phenylvaleric acid
}

\author{
I-Ching Ho ${ }^{a}$, Sheng-Pin Yang ${ }^{b}$, Wen-Yen Chiu ${ }^{a, b, c, *}$, Shih-Yow Huang ${ }^{a}$ \\ a Department of Chemical Engineering, National Taiwan University, Taipei 10617, Taiwan, ROC \\ ${ }^{\mathrm{b}}$ Department of Materials Science and Engineering, National Taiwan University, Taipei 10617, Taiwan, ROC \\ ${ }^{\mathrm{c}}$ Institute of Polymer Science and Engineering, National Taiwan University, Taipei 10617, Taiwan, ROC
}

Received 4 May 2006; received in revised form 14 June 2006; accepted 19 June 2006

Available online 27 June 2006

\begin{abstract}
PHAs (poly-3-hydroxyalkanoates) obtained by Pseudomonas oleovorans grown with mixed carbon sources were investigated. Mixed carbon sources were sodium octanoate/undecylenic acid and sodium octanoate/5-phenylvaleric acid. Effect of carbon source in pre-culture on PHAs structure was investigated. Main fermentation was conducted with mixture of sodium octanoate/undecylenic acid, and PHA contained both saturated and unsaturated units. When more undecylenic acid was used in the medium, the ratio of unsaturated unit increased and the $T_{\mathrm{g}}$ of the products also changed. The PHA grown with mixture of sodium octanoate and undecylenic acid was a random copolymer, which was determined by DSC analysis. Using mixed carbon sources of sodium octanoate and 5-phenylvaleric acid, highest dry cell weight and PHA concentration were obtained when $0.02 \mathrm{~g}$ or $0.04 \mathrm{~g}$ of 5-phenylvaleric acid were added in $50 \mathrm{~mL}$ medium. Cultured with sodium octanoate and 5-phenylvaleric acid, PHA containing HO (3-hydroxyoctanoate) unit and HPV (3-hydroxy-5-phenylvalerate) unit was produced. $T_{\mathrm{g}}$ of the products fell between those of pure PHO and pure PHPV. By means of DSC analysis and fractionation method, the PHA obtained was regarded as a random copolymer.
\end{abstract}

(c) 2006 Elsevier B.V. All rights reserved.

Keywords: Pseudomonas oleovorans; Polymer form; Undecylenic acid; 5-Phenylvaleric acid; Poly-3-hydroxyalkanoates

\section{Introduction}

PHAs (Poly-3-hydroxyalkanoates) are intracellular energy storage polyester supplied for bacteria. When carbon sources were used up, depolymerization of PHA induced inside the bacterium and PHA transferred to energy for living. Diameter of PHAs is about $0.1-0.7 \mu \mathrm{m}$ inside the cytoplasm of bacteria, and molecular weight of PHAs lays between 50,000 and $1,000,000 \mathrm{Da}$ [1]. At present, at least 75 different strains of bacteria, which could produce PHA were discovered and PHA content (g PHA/g dry cell weight) as high as $90 \%$ was identified. More than 100 different PHAs containing various side chain groups

\footnotetext{
* Corresponding author. Tel.: +88622362 3259; fax: +886223623040.

E-mail addresses: ycchiu@ccms.ntu.edu.tw, ycchiu@ntu.edu.tw (W.-Y. Chiu).
}

were confirmed [2]. PHAs are biodegradable thermoplastics [3] whose mechanical properties depended on their structure ranging from brittle to elastic [4].

PHAs were divided into two groups according to the number of carbons [5]. One was scl-PHAs (short-chain-length PHAs), which had three to five carbons. The other was mcl-PHAs (medium-chain-length PHAs), which contained 6-14 carbons. Pseudomonas oleovorans is one of bacteria, which could produce mcl-PHAs with different functional groups on the side chain. Many PHAs with various side chain types and side chain groups were reported, such as phenyl group [6,7], unsaturated side chain (olefin) [8,9], branched side chain [10], halogen containing side chains [11-13].

The main structure of PHA was PHO (poly-3-hydroxyoctanoate) when octane was used as sole carbon source by P. oleovorans [14]. Copolymer of three different monomers, 3-hydroxy-10-undecenoate, 3-hydroxy-8-noneneoate and 3- 
hydroxy-6-hepteneoate [9], and PHPV (poly-3-hydroxy-5phenylvalerate) which contained phenyl group [7] were obtained when undecylenic acid and 5-phenylvaleric acid were served as carbon source, respectively. PHA grown with two different carbon sources contained two different main structures. When dosing different ratio of mixed carbon sources, structures and physical properties of PHAs were affected $[9,15]$. Therefore, an efficient strategy to control structure and polymer form [9,15-18] would improve the applications of PHAs. Moreover, PHAs with unsaturated side chain were reactive biopolyesters, which could be used for chemical modification, such as crosslinking reaction, attachment of bioreactive substances [19], and hydrophilic modification [20-24]. Modifying PHAs with both saturated and unsaturated side chain groups, polyesters with both hydrophilic and hydrophobic functional groups were obtained. Hydrophilic/hydrophobic balance was adjusted through copolymer components. For example, Kurth et al. [20] used PHA containing hydrophilic and hydrophobic groups as carrier of drugs.

Few researchers focused on the effect of carbon sources of pre-culture. In this work, effect of carbon sources in pre-culture on PHAs structure was discussed. With different carbon sources for pre-culture and different ratio of mixed carbon sources (sodium octanoate/undecylenic acid and sodium octanoate/5phenylvaleric acid) for main fermentation, polymer structure was detected by NMR, $T_{\mathrm{g}}$ and polymer form were determined by DSC.

\section{Materials and methods}

\subsection{Microorganism and medium}

P. oleovorans ATCC 29347 purchased from Bioresource Collection and Research Center, Food Industry Research and Development Institute (Hsinchu, Taiwan) was used in this work. P. oleovorans was stored on nutrient agar plate. The medium contained (per liter): $6 \mathrm{~g}$ citric acid monohydrate, $4 \mathrm{~g}$ $\left(\mathrm{NH}_{4}\right)_{2} \mathrm{SO}_{4}, 5.8 \mathrm{~g} \mathrm{~K}_{2} \mathrm{HPO}_{4}, 3.7 \mathrm{~g} \mathrm{KH}_{2} \mathrm{PO}_{4}, 0.4 \mathrm{~g} \mathrm{MgSO} 4 \cdot 7 \mathrm{H}_{2} \mathrm{O}$, $1 \mathrm{~mL}$ microelement solution and $1.5 \mathrm{wt} \%$ agar. Microelement solution contained (per liter): $2.78 \mathrm{~g} \mathrm{FeSO}_{4} \cdot 7 \mathrm{H}_{2} \mathrm{O}, 1.98 \mathrm{~g}$ $\mathrm{MnCl}_{2} \cdot 4 \mathrm{H}_{2} \mathrm{O}, 2.81 \mathrm{~g} \mathrm{CoSO} 4 \cdot 7 \mathrm{H}_{2} \mathrm{O}, 1.67 \mathrm{~g} \mathrm{CaCl}_{2} \cdot 2 \mathrm{H}_{2} \mathrm{O}, 0.29 \mathrm{~g}$ $\mathrm{ZnSO}_{4} \cdot 7 \mathrm{H}_{2} \mathrm{O}$, and $1 \mathrm{M} \mathrm{HCl}$. The $\mathrm{pH}$ was adjusted to 7.5 with $2 \mathrm{~N} \mathrm{NaOH}$ before sterilization. The agar plate was cultured in $30^{\circ} \mathrm{C}$ incubator for 3 days and reserved at $4{ }^{\circ} \mathrm{C}$.

\subsection{Flask culture}

Pre-culture and main fermentation were performed in $250 \mathrm{~mL}$ Erlenmeyer flasks containing $50 \mathrm{~mL}$ aqueous medium. Except for the carbon source, the aqueous medium and microelement solution possessed the same composition as that contained in the medium used in agar plate. Three carbon sources were dosed in this work: sodium octanoate, undecylenic acid, and 5phenylvaleric acid. The concentration of carbon source was kept at $36.10 \mathrm{mM}$. Sodium octanoate was added before the medium was sterilized in autoclave. Undecylenic acid was subsequently added into the sterilized medium through filter (MF-Millipore
MCE Membrane, $0.22 \mu \mathrm{m})$. 5-Phenylvaleric acid which had been dissolved in ethanol $(0.32 \mathrm{~g}$ 5-phenylvaleric acid and $3 \mathrm{~mL}$ ethanol) was also added through filter. After certain time of preculture, $5 \mathrm{~mL}$ of pre-culture solution was removed to the main fermentation medium. Cells were incubated in a rotary incubator at $160 \mathrm{rpm}$ and $30^{\circ} \mathrm{C}$.

\subsection{Analytical methods}

Fifty milliliters of culture broth was centrifuged at $12,000 \mathrm{rpm}$ for $15 \mathrm{~min}$. The upper liquid was withdrawn and a little ethanol was added to remove residual carbon source. After centrifugation, the precipitate was lyophilized to a constant weight. Dry cell weight (DCW) was determined by weighing lyophilized sample.

PHA was extracted from the lyophilized cells in $80 \mathrm{~mL}$ chloroform for $3 \mathrm{~h}$. After filtering the mixture, chloroform was removed by using a rotary vacuum evaporator at $45^{\circ} \mathrm{C}$. PHA was purified by methanol for $24 \mathrm{~h}$. After removing the methanol, weight of PHA was determined gravimetrically.

The composition of PHA was determined by ${ }^{1} \mathrm{H}$ NMR spectroscopy. Samples were dissolved in D-chloroform $(10 \mathrm{mg} / \mathrm{mL})$. ${ }^{1} \mathrm{H}$ NMR spectroscopy was recorded on Bruker Avance FT NMR spectrometer operating at $500 \mathrm{MHz}$ and completed by Instrumentation Center of National Taiwan University.

Melting points $\left(T_{\mathrm{m}}\right)$, glass transition temperatures $\left(T_{\mathrm{g}}\right)$ and heat of fusion $(\Delta H)$ were recorded on a TA Instrument DSC. The weight of sample was about $6-10 \mathrm{mg}$ and the sample was heated from -70 to $120^{\circ} \mathrm{C}$ at a heating rate of $10^{\circ} \mathrm{C} / \mathrm{min}$ under $50 \mathrm{~mL} / \mathrm{min}$ of nitrogen purge. Data of $T_{\mathrm{g}}$ shown in this investigation were read from the second run, and data of $T_{\mathrm{m}}$ shown in this investigation from the first run.

\subsection{Fractionation analysis}

In order to distinguish the polymer form-copolymer or blend of homopolymers, fractionation analysis was used for PHA obtained from cell growth using sodium octanoate and 5phenylvaleric acid as mixed carbon sources. The produced PHA was added into a mixed solvent (methanol:chloroform $=1: 1$ $(\mathrm{v} / \mathrm{v}))$, stirred for dissolving, and then separation of upper solution and precipitation was conducted [16]. The separation process was repeated. Solvents of the upper solution and precipitation were removed by rotary vacuum evaporator, respectively. Each polymer component was determined by ${ }^{1} \mathrm{H}$ NMR.

\section{Results and discussion}

\subsection{PHA biosynthesized with sodium octanoate and undecylenic acid}

In this work, effect of carbon source of pre-culture and ratio of mixed carbon source on cell growth, PHA accumulation and PHA content were investigated. Sodium octanoate and undecylenic acid were used as sole carbon source individually for pre-culture. Mixed carbon source of sodium octanoate and undecylenic acid with molar ratio of $2: 1,1: 1$ and 1:2 was dosed 


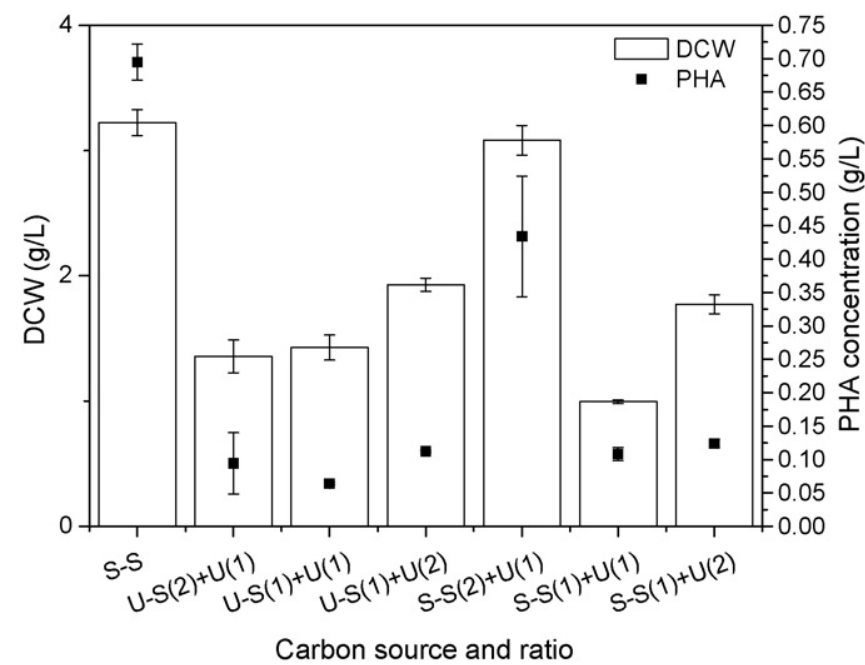

Fig. 1. Dry cell weight and PHA concentration produced by P. oleovorans grown with mixed carbon sources of sodium octanoate and undecylenic acid. $A-B(x)+C(y)$ represented carbon source of pre-culture was $A$ and carbon source of main fermentation was $B$ and $C$ with molar ratio of $x: y$. U: undecylenic acid; S: sodium octanoate.

respectively for main fermentation. By comparing the results obtained from mixed carbon source for main fermentation in Fig. 1, it shows that the dry cell weight (DCW) and PHA concentration reached the highest when dosing sodium octanoate for pre-culture, and dosing sodium octanoate/undecylenic acid with molar ratio of 2:1 for main fermentation.

Structure of PHA product was determined by ${ }^{1} \mathrm{H}$ NMR. Fig. 2 shows the structure of PHA produced by P. oleovorans grown with sodium octanoate and undecylenic acid, which includes structure 1, the HO (3-hydroxyoctanoate) unit, and structure 2, the HU (3-hydroxy-10-undecenoate-co-3-hydroxy8-noneneoate-co-3-hydroxy-6-hepteneoate) unit. Taking PHA

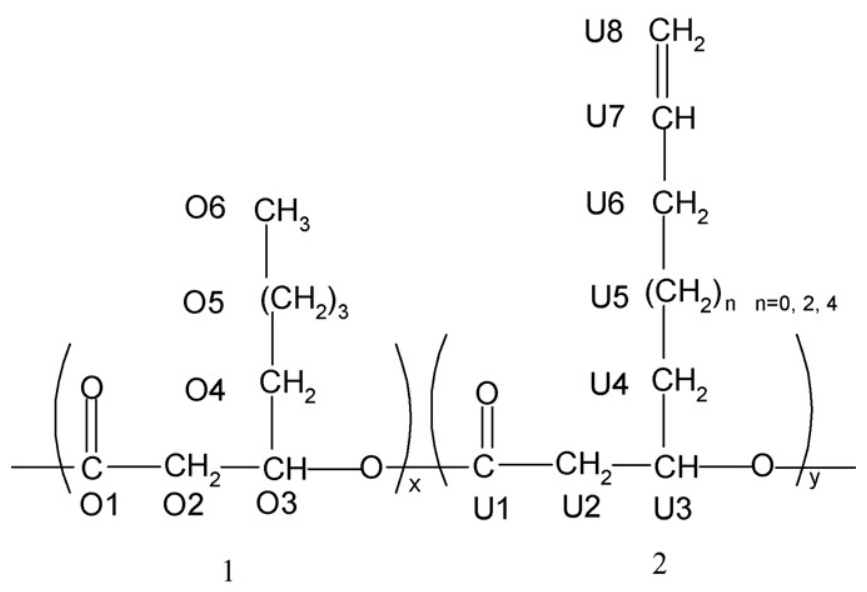

Fig. 2. Structure of PHA, which was obtained from sodium octanoate and undecylenic acid-grown cells.

biosynthesized with sodium octanoate in pre-culture and sodium octanoate/undecylenic acid with molar ratio of 2:1 in main fermentation, for example, its ${ }^{1} \mathrm{H}$ NMR spectroscopy was shown in Fig. 3. Obvious peaks at 4.9 and $5.7 \mathrm{ppm}$, which were positions of unsaturated unit of structure 2 (as shown in Fig. 2) revealed that the PHA included double bond. Saturated unit at the end of side chain in structure 1 (as shown in Fig. 2) was demonstrated by peak at $0.9 \mathrm{ppm}$. Position and area of each peak were collected in Table 1. Comparison between Figs. 2 and 3 and Table 1 revealed that each peak of structure was identical with ${ }^{1}$ H NMR spectroscopy. Kim et al. [9] reported that 3-hydroxy8-noneneoate was the main component of PHA biosynthesized with undecylenic acid. To simplify calculation, 3-hydroxy-8noneneoate was supposed as the only component of structure 2 in Fig. 2. Composition of PHA was determined from peak area at 0.9 and $5.7 \mathrm{ppm}$, which represented structures 1 and 2,

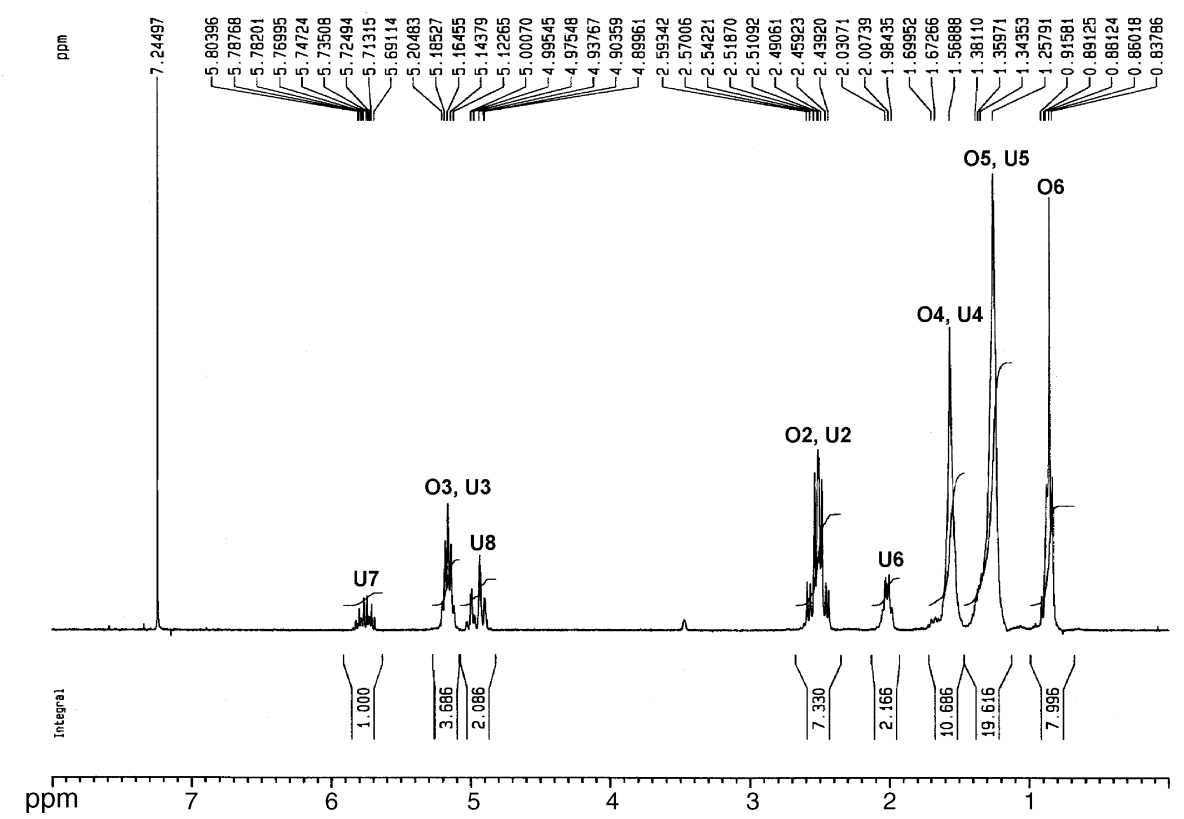

Fig. 3. ${ }^{1} \mathrm{H}$ NMR spectrum of PHA biosynthesized with mixed carbon source of sodium octanoate and undecylenic acid (carbon source of pre-culture was sodium octanoate and that of main fermentation was sodium octanoate/undecylenic acid with molar ratio of 2:1). 
Table 1

Chemical shifts (ppm) and peak area of PHA cultured with sodium octanoate and undecylenic acid (carbon source of pre-culture was sodium octanoate and that of main fermentation was sodium octanoate/undecylenic acid with molar ratio of 2:1)

\begin{tabular}{|c|c|c|c|c|c|c|c|c|}
\hline & \multicolumn{8}{|l|}{${ }^{1} \mathrm{H}$ NMR } \\
\hline & $\mathrm{O} 2, \mathrm{U} 2$ & O3, U3 & $\mathrm{O} 4, \mathrm{U} 4$ & O5, U5 & O6 & U6 & U7 & U8 \\
\hline Chemical shifts from literature ${ }^{a}$ & 2.5 & 5.2 & 1.6 & $1.26-1.36$ & 0.9 & 2.0 & 5.7 & 4.9 \\
\hline Chemical shifts from product & 2.5 & 5.2 & 1.6 & $1.26-1.38$ & 0.9 & 2.0 & 5.7 & 4.9 \\
\hline Number of hydrogen of structure $1^{b}$ & 2 & 1 & 2 & 6 & 3 & - & - & - \\
\hline Number of hydrogen of structure $2^{b}$ & 2 & 1 & 2 & 4 & - & 2 & 1 & 2 \\
\hline Peak area of PHA & 7.330 & 3.686 & 10.686 & 19.616 & 7.996 & 2.166 & 1.000 & 2.086 \\
\hline Calculated area of $\mathrm{PHA}^{\mathrm{c}}$ & 7.330 & 3.65 & 7.330 & 19.990 & 7.996 & 2.000 & 1.000 & 2.000 \\
\hline
\end{tabular}

${ }^{\text {a }}$ From $[9,25]$.

b See Fig. 2.

${ }^{\mathrm{c}}$ From peak area of O6 (0.9) and U7 (5.7), composition of structures 1 and 2 were 72.72 and 27.28 mol\%, respectively. Calculated area represented the area calculated by means of composition.

Table 2

$T_{\mathrm{g}}, T_{\mathrm{m}}$ and compositions of PHAs produced by P. oleovorans grown with different ratio of mixed carbon sources (sodium octanoate and undecylenic acid)

\begin{tabular}{|c|c|c|c|c|c|c|}
\hline $\begin{array}{l}\text { Carbon sources of pre-culture } \\
\text { and main fermentation }{ }^{\mathrm{a}}\end{array}$ & HO unit (mol\%) & HU unit (mol\%) & $T_{\mathrm{m}}\left({ }^{\circ} \mathrm{C}\right)$ & $T_{\mathrm{g}}\left({ }^{\circ} \mathrm{C}\right)$ & $T_{\mathrm{g}, \mathrm{F}}{ }^{\mathrm{b}}\left({ }^{\circ} \mathrm{C}\right)$ & $\Delta H(\mathrm{~J} / \mathrm{g})$ \\
\hline $\mathrm{U}-\mathrm{S}(2)+\mathrm{U}(1)$ & 36.27 & 63.73 & - & -45.23 & -42.34 & - \\
\hline $\mathrm{U}-\mathrm{S}(1)+\mathrm{U}(1)$ & 25.40 & 74.60 & - & - & - & - \\
\hline $\mathrm{U}-\mathrm{S}(1)+\mathrm{U}(2)$ & 26.91 & 73.09 & - & - & - & - \\
\hline $\mathrm{S}-\mathrm{S}(2)+\mathrm{U}(1)$ & 72.72 & 27.28 & - & -37.15 & -37.40 & - \\
\hline $\mathrm{S}-\mathrm{S}(1)+\mathrm{U}(1)$ & 50.84 & 49.16 & - & -43.42 & -40.22 & - \\
\hline $\mathrm{S}-\mathrm{S}(1)+\mathrm{U}(2)$ & 43.34 & 56.66 & - & - & - & - \\
\hline S-S & 100 & - & 58.40 & -34.40 & - & 19.71 \\
\hline U-U & 2 & 98 & - & -48.75 & - & - \\
\hline
\end{tabular}

${ }^{a} A-B(x)+C(y)$ represented carbon source of pre-culture was $A$ and carbon source of main fermentation was $B$ and $C$ with molar ratio of $x: y$. U: undecylenic acid; $\mathrm{S}$ : sodium octanoate.

${ }^{\mathrm{b}} T_{\mathrm{g}, \mathrm{F}}$ was calculated from Fox equation.

respectively. Calculated area represented the area calculated by means of composition. Table 1 shows that the calculated area was close to peak area except for the peak at $1.6 \mathrm{ppm}$. The error of peak at $1.6 \mathrm{ppm}$ came from the negligence of other possible structures in Fig. 2. Nevertheless, this result revealed that the assumption of structure 2 was acceptable and both structures 1 and 2 co-existed in the produced PHA.

The PHA composition calculated from peak area at 0.9 and $5.7 \mathrm{ppm}$ and its DSC analysis were shown in Table 2. PHA contained more unsaturated structure, HU unit when dosed with undecylenic acid as pre-culture carbon source. PHA composition changed with ratio of carbon sources used for main fermentation. Using higher ratio of saturated carbon source would produce $\mathrm{PHA}$ containing more saturated structure, $\mathrm{HO}$ unit, and vice versa. As a result, the composition of PHA product could be controlled by carbon source used in both pre-culture and main fermentation. From the result of DSC analysis, $T_{\mathrm{g}}$ of PHA biosynthesized with mixed carbon source fell between $-34.4^{\circ} \mathrm{C}$ ( $T_{\mathrm{g}}$ of PHA produced from sodium octanoate) and $-48.75^{\circ} \mathrm{C}$ ( $T_{\mathrm{g}}$ of PHA produced from undecylenic acid). No obvious peak of $T_{\mathrm{m}}$ appeared in DSC curve. PHA produced from undecylenic acid as sole carbon source had no $T_{\mathrm{m}}$, and $58.4^{\circ} \mathrm{C}$ of $T_{\mathrm{m}}$ was observed when PHA was produced from sodium octanoate as sole carbon source.

DSC analysis of a blend mixed with PHO (biosynthesized from sodium octanoate) and PHU (biosynthesized from unde- cylenic acid) was shown in Fig. 4. Two obvious shifts, -32.14 and $-44.5^{\circ} \mathrm{C}$, appeared in the DSC curve. Thus, blend of PHO and PHU had two $T_{\mathrm{g}}$. In addition, the melting peak was remarkably observed. Fig. 4 also shows DSC analysis of PHA produced from mixed carbon source, sodium octanoate and undecylenic

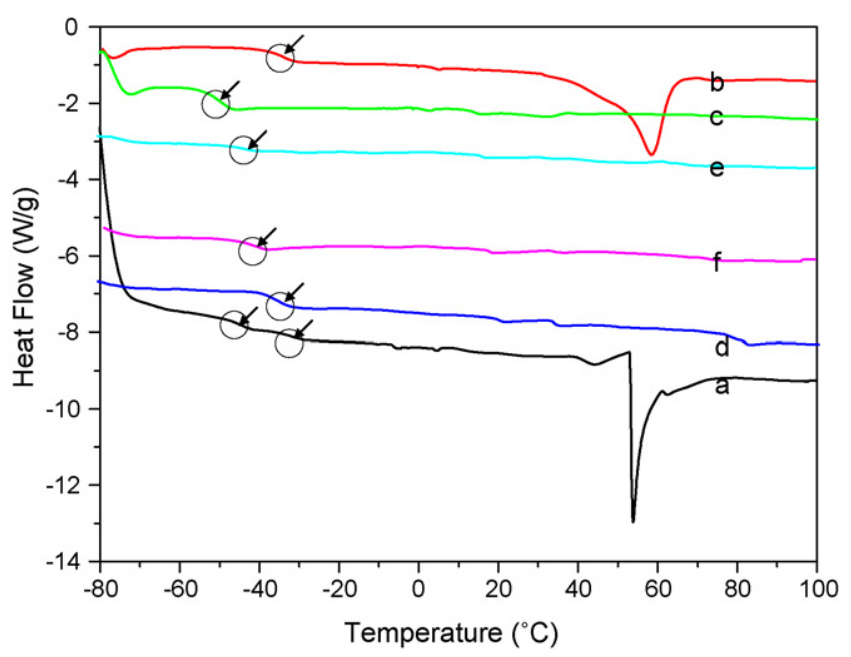

Fig. 4. DSC thermogram of PHA: (a) blend of $\mathrm{PHO}$ and $\mathrm{PHU}$ with weight ratio of 54:46; (b) PHO; (c) PHU; (d and e) PHAs produced from mixtures of SO and UA with molar ratio of 2:1 and 1:1 (pre-culture with SO); (f) PHAs produced from mixtures of SO and UA with molar ratio of 2:1 (pre-culture with UA). 
acid. When mixed carbon source was dosed, PHA possessed only one $T_{\mathrm{g}}$, which changed with composition of PHA and fell between $T_{\mathrm{g}}$ of PHO and $T_{\mathrm{g}}$ of PHU, and showed no peak of melting $\left(T_{\mathrm{m}}\right)$. Because of notable difference between blend of PHO/PHU and PHA from mixed carbon source, PHA biosynthesized with sodium octanoate and undecylenic acid could be a random copolymer. Here, Fox equation $1 / T_{\mathrm{g}}=w_{1} / T_{\mathrm{g}, 1}+w_{2} / T_{\mathrm{g}, 2}$ was used to estimate the $T_{\mathrm{g}}$ of random copolymers, where $T_{\mathrm{g}, 1}$, $T_{\mathrm{g}, 2}, w_{1}, w_{2}$ were represented $T_{\mathrm{g}}$ of pure PHO, $T_{\mathrm{g}}$ of pure $\mathrm{PHU}$, weight percentage of $\mathrm{HO}$ unit, and weight percentage of $\mathrm{HU}$ unit, respectively. From Table 2, experimental data conformed well with the calculated data from Fox equation. It demonstrated the product could be a random copolymer.

\subsection{PHA biosynthesized with sodium octanoate and 5-phenylvaleric acid}

In this part, carbon source of pre-culture was $0.75 \mathrm{~g} / \mathrm{L}$ of sodium octanoate, and $0.75 \mathrm{~g} / \mathrm{L}$ of sodium octanoate and different concentration of 5-phenylvaleric acid including $0.32,0.16$, $0.08,0.04$, and $0.02 \mathrm{~g} / 50 \mathrm{~mL}$ were dosed for main fermentation. Mixed carbon source including sodium octanoate was used to improve cell growth and PHA accumulation because P. oleovorans assimilated sodium octanoate more easily. Fig. 5 shows the dry cell weight (DCW) and PHA concentration biosynthesized with mixed carbon source of sodium octanoate and 5 -phenylvaleric acid. When a mixed carbon source of $0.75 \mathrm{~g} / \mathrm{L}$ sodium octanoate $/ 0.02 \mathrm{~g} / 50 \mathrm{~mL}$ 5-phenylvaleric acid was used, DCW and PHA were both higher than that produced from sodium octanoate used as sole carbon source. PHA concentration and PHA content were higher when 0.02 or $0.04 \mathrm{~g} / 50 \mathrm{~mL}$ of 5-phenylvaleric acid along with $0.75 \mathrm{~g} / \mathrm{L}$ of sodium octanoate were dosed than that with dosing sodium octanoate merely.

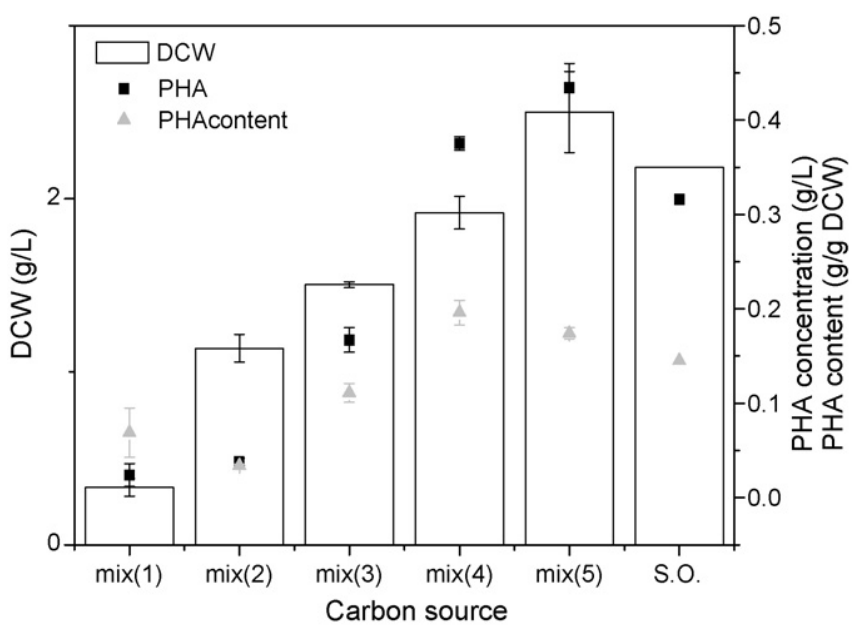

Fig. 5. Dry cell weight, PHA concentration and PHA content produced by P. oleovorans grown with mixed carbon sources of sodium octanoate and 5phenylvaleric acid. Mix(1): grown with $0.75 \mathrm{~g} / \mathrm{L}$ of $\mathrm{SO}$ and $0.32 \mathrm{~g} / 50 \mathrm{~mL}$ of 5-PVA; $\mathrm{Mix}(2)$ : grown with $0.75 \mathrm{~g} / \mathrm{L}$ of $\mathrm{SO}$ and $0.16 \mathrm{~g} / 50 \mathrm{~mL}$ of 5-PVA; $\mathrm{Mix}(3)$ : grown with $0.75 \mathrm{~g} / \mathrm{L}$ of $\mathrm{SO}$ and $0.08 \mathrm{~g} / 50 \mathrm{~mL}$ of 5-PVA; $\operatorname{Mix}(4)$ : grown with $0.75 \mathrm{~g} / \mathrm{L}$ of $\mathrm{SO}$ and $0.04 \mathrm{~g} / 50 \mathrm{~mL}$ of 5 -PVA; $\mathrm{Mix}(5)$ : grown with $0.75 \mathrm{~g} / \mathrm{L}$ of $\mathrm{SO}$ and $0.02 \mathrm{~g} / 50 \mathrm{~mL}$ of 5-PVA; S.O.: grown with $0.75 \mathrm{~g} / \mathrm{L}$ of SO; SO: sodium octanoate; 5-PVA: 5-phenylvaleric acid.

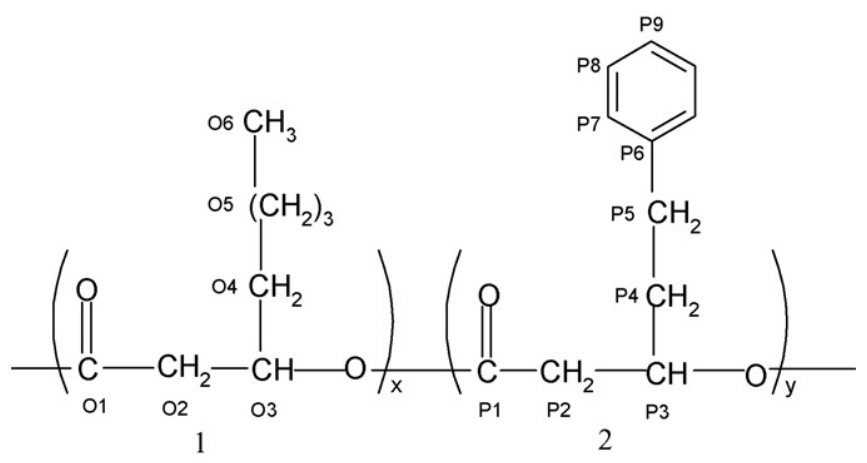

Fig. 6. Structure of PHA, which was obtained from sodium octanoate and undecylenic acid-grown cells.

Fig. 6 shows the structure of PHA produced by P. oleovorans grown with sodium octanoate and 5-phenylvaleric acid. ${ }^{1} \mathrm{H}$ NMR spectroscopy of PHA, which was biosynthesized with sodium octanoate for pre-culture and $0.75 \mathrm{~g} / \mathrm{L}$ sodium octanoate $/ 0.16 \mathrm{~g} / 50 \mathrm{~mL} 5$-phenylvaleric acid for main fermentation, was shown in Fig. 7. Each peak of prediction structure (Fig. 6) and ${ }^{1} \mathrm{H}$ NMR spectroscopy (Table 3 and Fig. 7) were identical. Peaks at around 7.1-7.2 and $1.9 \mathrm{ppm}$ revealed that PHA contained phenyl group as structure 2, the HPV (3hydroxy-5-phenylvalerate) unit of Fig. 6. Peak at 0.9 ppm, which represented saturated unit at the end of side chain as structure 1, the HO unit of Fig. 6, appeared in PHA. Composition of PHA was determined from peak area at 0.9 and $1.9 \mathrm{ppm}$ listed in Table 3. It shows that the calculated area was close to peak area except for the peak at $1.6 \mathrm{ppm}$. The error of peak at $1.6 \mathrm{ppm}$ came from the negligence of other possible structures in structure 1 of Fig. 6. The overall coincidence between the peak area and calculated area represented that PHA mainly contained two structures, HO unit and HPV unit. In this work, a small amount of ethanol was used as co-solvent to increase the solubility of 5-phenylvaleric acid. No new peak in Fig. 7 was found. It shows that no new structure was produced. According to the experimental results by Sanguanchaipaiwong et al. [26], the addition of diethylene glycol in the medium would change the composition of PHA toward the 3-hydroxyoctanoate. Ethanol was expected to have the same effect on the change of composition of PHA which allowing the assumption that the only composition in structure 1 of Fig. 6 to be more reasonable.

Composition of PHA and its DSC analysis were shown in Table 4. Ratio of two structures changed with concentration of 5-phenylvaleric acid. Among them, the ratio of HPV unit was highest when $0.75 \mathrm{~g} / \mathrm{L}$ of sodium octanoate and $0.16 \mathrm{~g} / 50 \mathrm{~mL} 5$ phenylvaleric acid were dosed as mixed carbon source. According to DSC data, $T_{\mathrm{g}}$ and $T_{\mathrm{m}}$ of PHA produced from mixed carbon source were all higher than that of PHO. Because $T_{\mathrm{g}}$ and $T_{\mathrm{m}}$ of pure PHPV were both higher than those of PHO, the above results were reasonable. No obvious difference was found between $\triangle H$ of PHA biosynthesized with mixed carbon source and $\Delta H$ of PHO because product contained only small fraction of HPV unit.

From Table $4, T_{\mathrm{g}}$ of PHA biosynthesized with sodium octanoate/5-phenylvaleric acid fell between $-34.4{ }^{\circ} \mathrm{C}\left(T_{\mathrm{g}}\right.$ of 


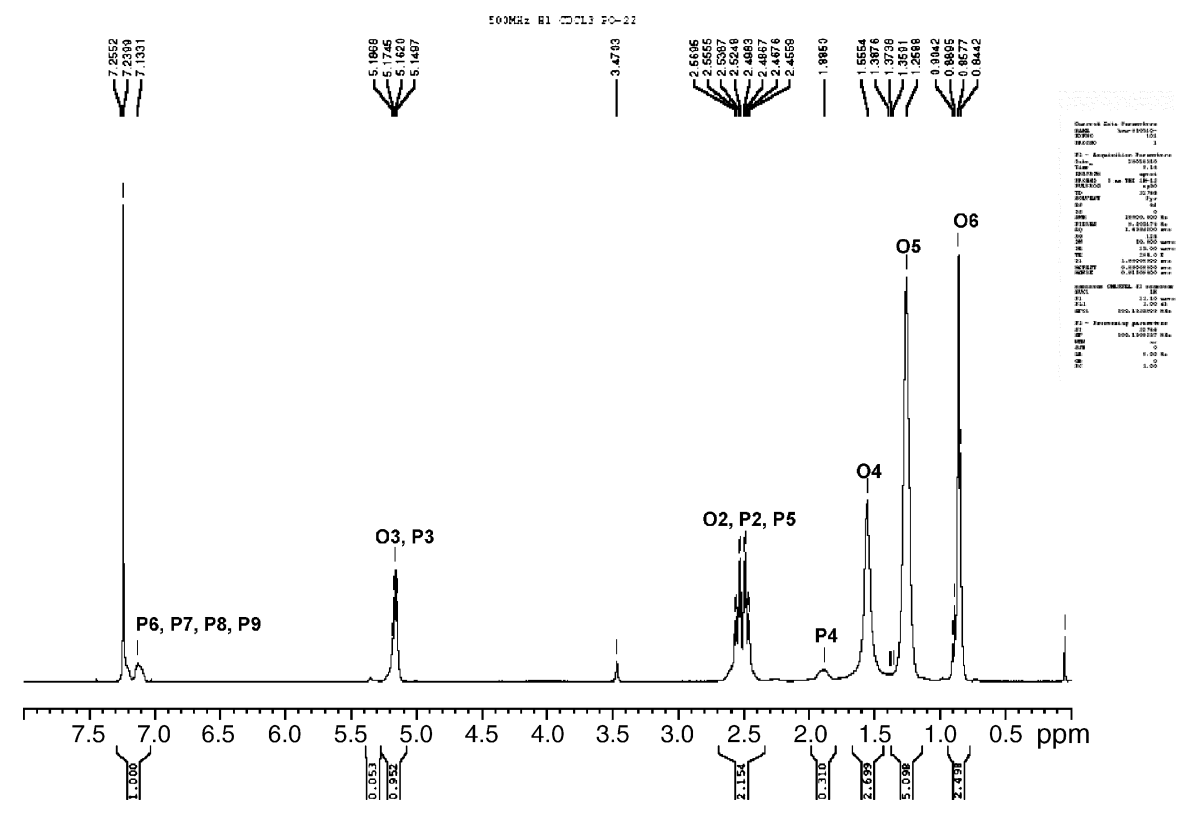

Fig. 7. ${ }^{1} \mathrm{H}$ NMR spectrum of PHA biosynthesized with mixed carbon source of sodium octanoate and 5-phenylvaleric acid (carbon source of pre-culture was $0.75 \mathrm{~g} / \mathrm{L}$ of sodium octanoate and that of main fermentation was $0.75 \mathrm{~g} / \mathrm{L}$ of sodium octanoate $/ 0.16 \mathrm{~g} / 50 \mathrm{~mL}$ of 5 -phenylvaleric acid).

Table 3

Chemical shifts (ppm) and peak area of PHA cultured with sodium octanoate and 5-phenylvaleric acid (carbon source of pre-culture was $0.75 \mathrm{~g} / \mathrm{L}$ of sodium octanoate and that of main fermentation was $0.75 \mathrm{~g} / \mathrm{L}$ of sodium octanoate $/ 0.16 \mathrm{~g} / 50 \mathrm{~mL}$ of 5 -phenylvaleric acid)

\begin{tabular}{|c|c|c|c|c|c|c|c|}
\hline & \multicolumn{7}{|c|}{${ }^{1} \mathrm{H}$ NMR } \\
\hline & O6 & O5 & $\mathrm{O} 4$ & P4 & $\mathrm{O} 2, \mathrm{P} 2, \mathrm{P} 5$ & $\mathrm{O} 3, \mathrm{P} 3$ & P6, P7, P8, P9 \\
\hline Chemical shifts from literature $^{a}$ & 0.9 & 1.2 & 1.6 & 1.9 & $2.5-2.6$ & 5.2 & $7.14-7.22$ \\
\hline Chemical shifts from product & 0.9 & 1.2 & 1.6 & 1.9 & $2.5-2.6$ & 5.2 & $7.13-7.25$ \\
\hline Number of hydrogen of structure $1^{b}$ & 3 & 6 & 2 & - & 2 & 1 & - \\
\hline Number of hydrogen of structure $2^{b}$ & - & - & - & 2 & 4 & 1 & 5 \\
\hline Peak area of PHA & 2.498 & 5.098 & 2.699 & 0.310 & 2.154 & 0.952 & $<1.000$ \\
\hline Calculated area of $\mathrm{PHA}^{\mathrm{c}}$ & 2.498 & 4.9962 & 1.665 & 0.310 & 2.285 & 0.9877 & 0.775 \\
\hline
\end{tabular}

${ }^{a}$ From $[7,25]$.

b See Fig. 6.

${ }^{c}$ From peak area of O6 (0.9) and P4 (1.9), composition of structures 1 and 2 were 84.3 and 15.7 mol\%, respectively. Calculated area represented the area calculated by means of composition.

PHA produced from sodium octanoate) and $13^{\circ} \mathrm{C}\left(T_{\mathrm{g}}\right.$ of PHA produced from 5-phenylvaleric acid) and changed with the composition of the product. Therefore, the PHA produced with mixed carbon source was supposed to be a random copolymer. Chung et al. [16] reported that the polymer form-copolymer or blend of homopolymers of PHA obtained from bacteria grown with sodium octanoate and 5-phenylvaleric acid could be examined by fractionation analysis. The PHA was added into a mixed solvent (methanol:chloroform $=1: 1(\mathrm{v} / \mathrm{v})$ ), and it was separated into upper solution and precipitation. Composition of each part of PHA was determined by ${ }^{1} \mathrm{H}$ NMR. As shown in Fig. 8, a sample of PHA containing $8.1 \mathrm{~mol} \%$ of HPV unit was under a

Table 4

$T_{\mathrm{g}}, T_{\mathrm{m}}, \Delta H$ and compositions of PHAs produced by P. oleovorans grown with different ratio of mixed carbon sources (sodium octanoate and 5-phenylvaleric acid)

\begin{tabular}{|c|c|c|c|c|c|c|}
\hline Carbon sources of pre-culture and main fermentation ${ }^{\mathrm{a}}$ & HPV unit ${ }^{\mathrm{b}}(\mathrm{mol} \%)$ & HO unit ${ }^{b}(\mathrm{~mol} \%)$ & Heating rate $\left({ }^{\circ} \mathrm{C} / \mathrm{min}\right)$ & $T_{\mathrm{m}}\left({ }^{\circ} \mathrm{C}\right)$ & $T_{\mathrm{g}}\left({ }^{\circ} \mathrm{C}\right)$ & $\Delta H(\mathrm{~J} / \mathrm{g})$ \\
\hline $\mathrm{SO} 0.75 \mathrm{~g} / \mathrm{L} \rightarrow \mathrm{SO} 0.75 \mathrm{~g} / \mathrm{L}+5 \mathrm{PVA} 0.16 \mathrm{~g} / 50 \mathrm{~mL}$ & 15.7 & 84.3 & - & - & - & - \\
\hline $\mathrm{SO} 0.75 \mathrm{~g} / \mathrm{L} \rightarrow \mathrm{SO} 0.75 \mathrm{~g} / \mathrm{L}+5$ PVA $0.08 \mathrm{~g} / 50 \mathrm{~mL}$ & 9.6 & 90.4 & 10 & 61.12 & -30.61 & 17.57 \\
\hline $\mathrm{SO} 0.75 \mathrm{~g} / \mathrm{L} \rightarrow \mathrm{SO} 0.75 \mathrm{~g} / \mathrm{L}+5 \mathrm{PVA} 0.04 \mathrm{~g} / 50 \mathrm{~mL}$ & 10.4 & 89.6 & 10 & 62.16 & -32.73 & 21.68 \\
\hline $\mathrm{SO} 0.75 \mathrm{~g} / \mathrm{L} \rightarrow \mathrm{SO} 0.75 \mathrm{~g} / \mathrm{L}+5 \mathrm{PVA} 0.02 \mathrm{~g} / 50 \mathrm{~mL}$ & 8.1 & 91.9 & 10 & 62.84 & -31.63 & 18.67 \\
\hline $5 \mathrm{PVA} \rightarrow 5 \mathrm{PVA}^{\mathrm{c}}$ & 100 & - & 20 & 66 & 13 & 1.5 \\
\hline $\mathrm{SO} \rightarrow \mathrm{SO}$ & - & 100 & 10 & 58.4 & -34.4 & 19.71 \\
\hline
\end{tabular}

${ }^{\mathrm{a}} A \rightarrow B$ represented carbon source of pre-culture was $A$ and carbon source of main fermentation was $B$. SO: sodium octanoate; 5PVA: 5-phenylvaleric acid.

${ }^{\mathrm{b}}$ HPV unit: 3-hydroxy-5-phenylvalerate; HO unit: 3-hydroxyoctanoate

${ }^{\mathrm{c}}$ From [9]. 


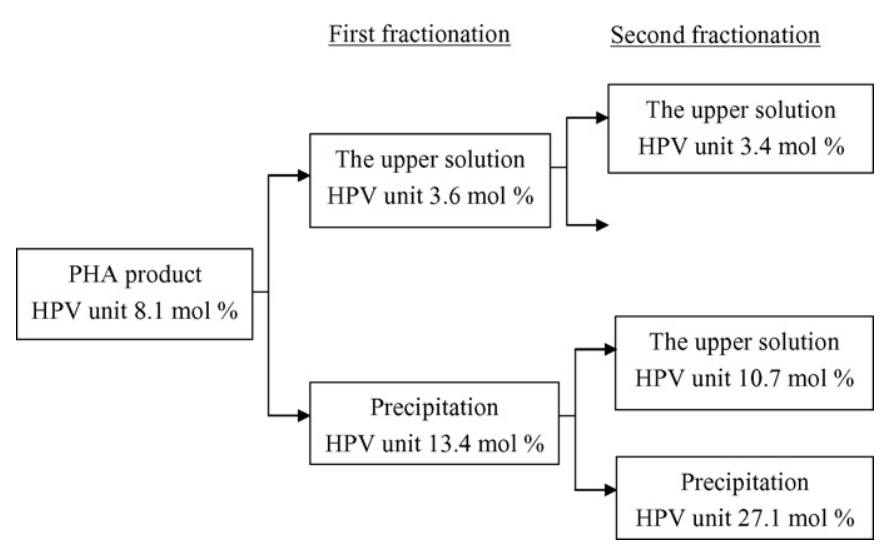

Fig. 8. Composition of PHA biosynthesized with sodium octanoate and 5phenylvaleric acid and its separation composition.

process of fractionation. After first separation, obtained from upper solution the PHA contained $3.6 \mathrm{~mol} \% \mathrm{HPV}$ unit and the PHA from precipitation comprised $13.4 \mathrm{~mol} \%$ HPV unit. The upper solution was separated secondly, ratio of HPV unit slightly decreased in the second separation of upper solution, but pure PHO could not be obtained. The precipitation of first separation was also separated, the upper solution of second separation contained $10.7 \mathrm{~mol} \% \mathrm{HPV}$ unit and the precipitation of second separation included $27.1 \mathrm{~mol} \% \mathrm{HPV}$ unit. Even though the ratio of HPV unit in the precipitation of second separation increased, pure PHPV cannot be gained through repeated separation. The PHA biosynthesized with sodium octanoate/5phenylvaleric acid was confirmed as a random copolymer. Given the nature of random copolymer, the precipitation contained both HPV and HO units. Otherwise, PHO should dissolve in the upper solution completely.

\section{Conclusions}

Mixed carbon source was used in this investigation. Composition, $T_{\mathrm{g}}$ and polymer form of PHA produced by P. oleovorans grown with different carbon sources for pre-culture and main fermentation were discussed. When sodium octanoate and undecylenic acid were used as a mixed carbon source for main fermentation, the composition of PHA would change with carbon source of pre-culture and the ratio of mixed carbon source of main fermentation. The more undecylenic acid used, the more unsaturated units were obtained in the product. When sodium octanoate and 5-phenylvaleric acid were dosed as mixed carbon source, the ratio of HPV unit increased with the increase of concentration of 5-phenylvaleric acid. From DSC analysis, random copolymers were suggested no matter what mixed carbon source was used, such as sodium octanoate/undecylenic acid and sodium octanoate/5-phenylenic acid.

\section{Acknowledgement}

The authors are grateful for financial support from the National Science Council of ROC (grant no.: NSC 93-2621Z-002-026).

\section{References}

[1] L.L. Madison, G.W. Huisman, Microbiol. Mol. Biol. Rev. 63 (1999) 21.

[2] C.S.K. Reddy, R. Ghai, V. Rashmi, C. Kalia, Bioresource Technol. 87 (2003) 137.

[3] E. Grothe, M.M. Young, Y. Chisti, Enzyme Microb. Technol. 25 (1999) 132 .

[4] G.Q. Chen, Q. Wu, K. Zhao, P.H. Yu, Chin. J. Polym. Sci. 18 (2000) 389.

[5] S.Y. Lee, Biotechnol. Bioeng. 49 (1996) 1.

[6] S.M. Arestegui, M.A. Aponte, E. Diaz, E. schroder, Macromolecules 32 (1999) 2889.

[7] K. Fritzsche, R.W. Lenz, R.C. Fuller, Makromolekulare Chem. 191 (1990) 1957.

[8] A. Ballistreri, G. Montaudo, M. Giuffrida, R.W. Lenz, Y.B. Kim, R.C. Fuller, Macromolecules 25 (1992) 1845.

[9] Y.B. Kim, R.W. Lenz, R.C. Fuller, J. Polym. Sci. A, Polym. Chem. 33 (1995) 1367.

[10] B. Hazer, R.W. Lenz, R.C. Fuller, Macromolecules 27 (1994) 45.

[11] Y. Doi, C. Abe, Macromolecules 23 (1990) 3705.

[12] Y.B. Kim, R.W. Lenz, R.C. Fuller, Macromolecules 25 (1992) 1852.

[13] O.R. Kim, A. Gross, W.J. Hammar, R.A. Newmark, Macromolecules 29 (1996) 4572.

[14] M.J. de Smet, G. Eggink, B. Witholt, J. Kingma, H. Wynberg, J. Bacteriol. 154 (1983) 870.

[15] Y.B. Kim, R.W. Lenz, R.C. Fuller, Macromolecules 24 (1991) 5256.

[16] D.M. Chung, M.H. Choi, J.J. Song, S.C. Yoon, I.-K. Kang, N.E. Huh, Int. J. Biol. Macromol. 29 (2001) 243.

[17] J.M. Curley, R.W. Lenz, R.C. Fuller, Int. J. Biol. Macromol. 19 (1996) 29.

[18] Y.B. Kim, Y.-H. Rhee, R.W. Lenz, R.C. Fuller, Polym. J. 29 (1997) 894.

[19] M.M. Bear, M.-A. Leboucher-Durand, V. Langlois, R.W. Lenz, S. Goodwin, P. Guerin, React. Funct. Polym. 34 (1997) 65.

[20] N. Kurth, E. Renard, F. Brachet, D. Robic, Ph. Guerin, R. Bourbouze, Polymer 43 (2002) 1095.

[21] M.Y. Lee, W.H. Park, Macromol. Chem. Phys. 201 (2000) 2771.

[22] M.Y. Lee, W.H. Park, R.W. Lenz, Polymer 41 (2000) 1703.

[23] E. Renard, A. Poux, L. Timbart, V. Langlois, P. Guerin, Biomacromolecules 6 (2005) 891.

[24] D. Strigers, G.N. Tew, Biomacromolecules 4 (2003) 195.

[25] R.A. Gross, C. de Mello, R.W. Lenz, Macromolecules 22 (1989) 1106.

[26] V. Sanguanchaipaiwong, C.L. Gabelish, J. Hook, C. Scholz, L.J. Foster, Biomacromolecules 56 (2004) 43. 